\title{
TOPOLOGY OPTIMIZATION OF ACTIVE POWER FILTERS
}

\author{
Raul Monteiro ${ }^{1,2}$, Beatriz Borges ${ }^{1,3}$, Victor Anunciada ${ }^{1,3}$ \\ ${ }^{1}$ Instituto de Telecomunicações \\ Polo de Lisboa, IST Torre Norte \\ 1049-001 Lisboa, Portugal \\ phone: +351218418 385, fax: +351218418 472, e-mail: avaa@1x.it.pt, bborges@1x.it.pt \\ ${ }^{2}$ Instituto Politécnico de Tomar \\ Estrada da Serra, Tomar, Portugal \\ e-mail:raulm@ipt.pt \\ ${ }^{3}$ Instituto Superior Técnico \\ 1049-001 Lisboa, Portugal
}

\begin{abstract}
Active power filters (APF) are an important research domain, since several years ago. However the industrial use of APF is almost inexistent due to their high acquisition cost and to the absence of widely accepted criteria for usefulness. In most situations, where APF would be adopted, the plant management prefers a passive filter solution, considering simplicity, cost and rudeness. Consequently, power filtering is good for the mains network but presents a limited interest for the plant manager. Power quality in the user plant must be the main objective of active filters, instead of the suppression of harmonic content in the mains currents. This one must be a secondary objective, also achieved, but less important for the plant management, unless the contract with the power company obliges the reduction of current harmonic distortion. This approach is required to obtain the economic viability of active power filters. Consequently, the main economic objective of power filtering must be the power quality in the user plant. The power quality parameters can be evaluated as factors of cost in the design of active power filters. Another concern on the design and use of active power filters is the problem of the radio frequency conducted noise induced in the mains network. The hard switching technology, for bridge power converters, has been replaced, in our design of APF, by soft-switching technology. This paper presents a new design approach of APF based on the association of full-bridge hf soft-switching DC-DC converter with 6 cycloconverters (integral cycle control).
\end{abstract}

Key words:

Active power filters, power quality, soft-switching converters

\section{Introduction}

Shunt active power filters are converters connected in parallel with mains network. (Fig. 1). The power circuit is typically a 3-phase bridge converter, with hard switched IGBTs and an energy storage capacitor. In the last few years, a great amount of research work has been developed concerning the control methods of the bridge converter.

The challenging control problem is to achieve an ideal behavior characterized by:
- sinusoidal line currents, independent of the harmonic distortion of load currents

- to keep the storage capacitor voltage within an acceptable range, implying a very accurate control of the active power at the output of the converter; this condition means that the average output power of the active filter, during each line frequency period, must be maintained at a very low value (theoretically zero) to avoid the transfer of a large amount of energy to the storage capacitor;

- to achieve the previous targets even in the presence of hard transient conditions of load currents and, or, input voltage.

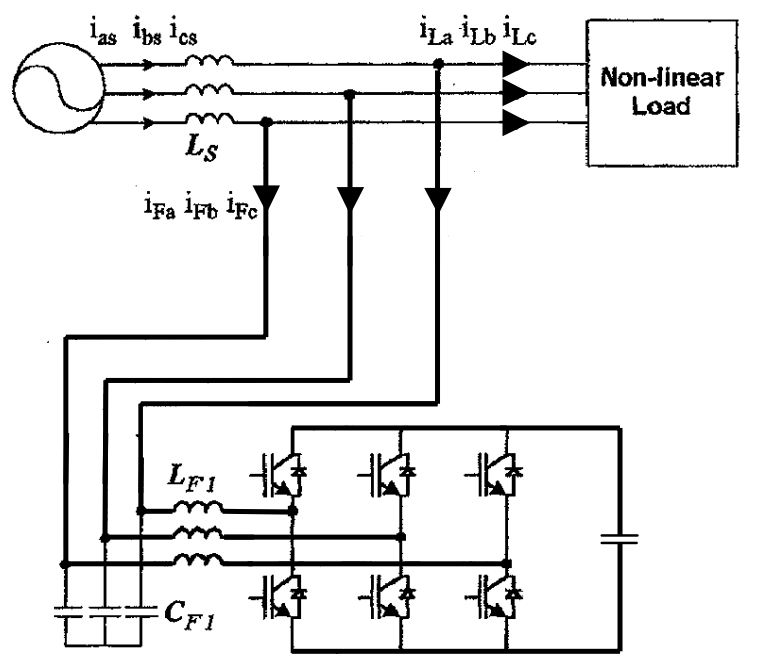

Fig.1. Shunt power active filter

Besides a control problem, this is also a feasibility problem of active power filters, because the accuracy and the time response of the control are very important to keep volume and cost of the capacitor at an acceptable level. In fact, it is difficult, in transient operating conditions, to avoid the transfer of a large amount of 
energy to the capacitor, or from the capacitor, during one period of line voltage. When a significant energy variation occurs, the capacitor voltage varies several hundred volt, even if the storage capacitance is very high.

Several authors use prediction computational methods to evaluate the amplitudes and phases of the fundamental current and first higher harmonics. This is the usual technical approach to perform an accurate control of the output current, assuring that average output power of the converter is zero [1]. Although this is a good approach in steady state operation, it fails in transient conditions. Even an urge evolution of those algorithms does not solve the problem in fast transient situations and in the presence of several loads, as electro-erosion and arc furnaces, where currents are stochastic, with a continuous spectrum [2]. Finally, the shunt active filters present two important drawbacks. The first one is the high frequency noise conducted to the power network, due to the hard switching IGBT circuits. It is not an easy and costless work to reduce the high frequency noise, in the converter output, into the maximum level of the european standard, namely in the frequency band of $1-10 \mathrm{MHz}$. The second drawback is the high manufacturing cost of the active power filter, associated to a limited benefit to the user. In fact, the power converter of a shunt active power filter must be designed considering the instantaneous value of the output power. In the presence of highly distorted load currents, or when power factor correction is desired, the converter power rating must be equal to the power rating of the user plant (or maximum load power rating). The manufacturing cost of the converter is always larger than $150 € / \mathrm{KVA}$ for high power levels. The benefits for the user are not valuable, because the active filter does not protect his plant against any power quality problems. The filter is only interesting to the power company management. And they may oblige the use of a harmonic filter, but cannot specify the use of an active filter. Consequently, these filters remain an interesting academic subject without any industrial impact. In fact, it is not economically acceptable that an active power filter may cost almost the same as an UPS system (excluding batteries), or even more, without providing any protection to the user plant.

The use of power active filters may reach a bigger impact if the devices include power protection features that are useful and valuable to the user plant.

The main power quality problems that cause hard failures in the commercial and industrial equipments are:

- harmonic distortion on the line voltage, implying overheating in power supplies and power factor compensation capacitors;

- transient overvoltages, including high frequency oscillations due to load switching or capacitor switching, implying semiconductor disruptive phenomena, and also errors on magnetic data storage devices;

- voltage sags, producing also semiconductor disruptive phenomena, when high voltage time derivatives occur.
The resulting failures in equipments are economically important and, justify the use of large UPS systems very often. Usually sensitive and expensive loads are protected with isolated UPSs, even when power failure or long interruptions are acceptable. However, the costs resulting from the MTBF reduction of non-sensitive devices is not evaluated.

The shunt power active filter does not provide any protection to the referred power quality problems. The authors considered that industrial development of active power filters depends on the reduction of the manufacturing cost and on the change of the objectives, including load protection features.

In this paper we will show the feasibility of active power filters based on two controlled current generators per phase, designed for maximum power ratings that are a small part of the plant power rating. The current generators are high frequency AC-AC converters (integral cycle, single phase cycloconverters).

A single soft-switching DC-AC power converter, providing a trapezoidal high frequency carrier, drives the six current generators of a 3-phase filter. The output converters modulate this high frequency voltage, in order to provide the referred controlled current generators.

\section{Series - Parallel Filter Topologies}

Considering the two main objectives for power filtering:

- power quality in the user plant, assumed as a perfect sinusoidal waveform, almost independent of load current;

- harmonic content reduction in the line current;

it is necessary to include some impedance between the supply and the user plant, not necessarily provided by the plant input line and transformer. An input reactance is the less expensive way to correct the line voltage waveform, using the active circuitry of the APF, with an affordable generation of peak power in the current generator (fig.2).

The power factor compensation determines the need of a switched bank of capacitors. Both impedances perform a LC low-pass filter, with a variable cut-off frequency, depending on the load reactive power.

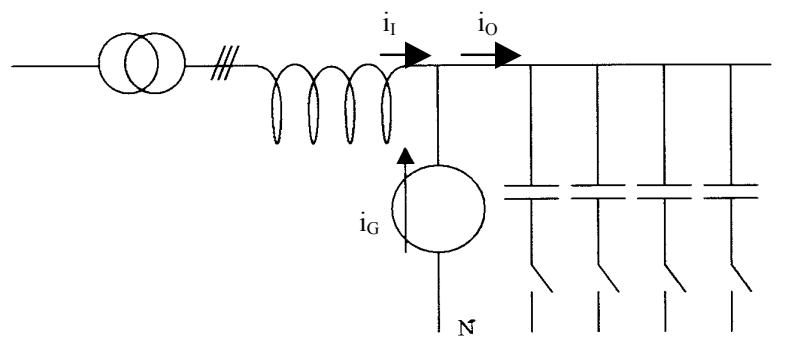

Fig. 2. Series-parallel PAF with input reactance 
The function of the current generator is to perform the short circuit of all harmonics produced in the load and to inject in the load the required current to keep voltage regulated and sinusoidal. The main design problem is to minimize the maximum instantaneous value of the output power of this current generator. A second design problem is to guarantee the system stability, considering the variation of the poles frequency. The first problem is a typical linear programming problem, where the frontier conditions result from the following variables:

- maximum allowed difference of voltages in mains network and in the user plant

- minimum allowed capacitive power factor

- minimum load power factor, without compensation, and the number of capacitor switches

- maximum value of the instantaneous value of the distortion current in the load

and cost functions are the manufacturing costs and acquisition costs versus peak instantaneous power of input inductances and current generators. The stability problem is quite complex and may be solved by the control circuitry of the current generator. This is a good solution, already adopted in the past by static interactive and rotative UPS manufacturers.

It is possible to show that the current generator controls the output voltage imposing to the output node a current equal to the sum of the following components [3]:

- reactive component of the load current

- all harmonics of the load current

This is true if the output voltage has a phase lag depending on the input current (Fig.3). Being this one approximately equal to the active component of the load current.

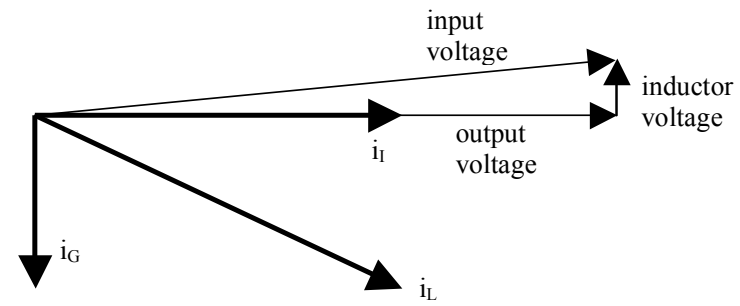

Fig. 3. Voltages and currents in the output node of a seriesparallel active filter (1st. harmonic)

When a switched capacitor bank compensates the load reactive power, the first harmonic of the current generator $\mathrm{i}_{\mathrm{G}}$ is approximately zero. Consequently, the current generator has a total current almost equal to the distortion components. The inductor voltage varies linearly with the input current and voltage phase lag depends on the inductor voltage. The phase lag of the output voltage must be controlled accurately because it is necessary to maintain the input power equal to the output power, unless the remaining energy will charge the input storage capacitor of the current generator. In a 3-phase system it is necessary to keep equal phase lags, in order to maintain a phase difference of 120 degrees, even when loads are different. The phase control remains complex and the input currents in the 3 phases would be equal. As the output currents are different and it is necessary to maintain the vectorial sum of the currents in the output nodes, the current generator will have an important active role.

As the reduction of the output power rating of the current generator is an important objective, the use of a passive constant inductance is not acceptable.

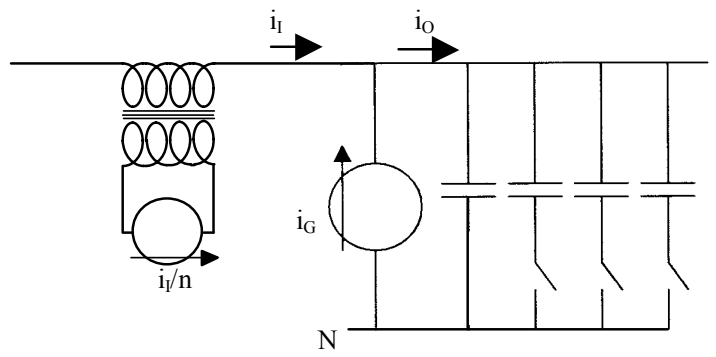

Fig. 4. Series-parallel PAF with two current generators

The replacement of the input inductance by a second current generator permits the independent regulation of the input currents, accordingly to each phase load. The power rating of this current generator is the product of the maximum peak input current by the instantaneous maximum difference of the input and output voltages. For power networks with high harmonic distortion levels, the referred voltage difference is usually less than $20 \%$ of the nominal input voltage. Consequently, this converter can be rated for $10 \%$ up to $20 \%$ of the plant rated power.

The control function of the input current generator performs the following functions:

- imposes an almost sinusoidal waveform for the input current;

- controls the current amplitude and phase in order to be equal to the first harmonic of the load current, with power factor compensation;

- adjusts the input current amplitude in order to control the voltage of the storage capacitor of the $\mathrm{DC}$-AC high frequency converter (active input power equal to the active output power condition);

As the input and load currents first harmonics (fundamental) are equal, the parallel current generator conducts only the distortion components of the load current. However, the parallel current generator and the power factor correction capacitors share those components. Consequently, the power rating of the current generator is lower than the product of the nominal voltage by the maximum peak value of distortion component. The critical harmonic for the design of the parallel current generator is the third harmonic, because the capacitors impedances decrease with the harmonic order. A good solution for filter cost reduction is to 
accept a leading input current, i.e., a minimum compensation capacitance, even in the absence of inductive loads.

The control function of the parallel current generator is to regulate the output voltage in order to obtain the same amplitude of the first harmonic of the input voltage, in phase.

It is important to note that control of series and parallel current generators are independent. Also input current and output voltage control of the 3 phases are completely independent.

\section{Cycloconverters driven by a Single h.f. Soft-Switching DC-AC Converter}

The six current generators, related with the 3 phases, are independent and independently controlled. Each parallel current generator is a cycloconverter supplied by the secondary of an hf transformer where a trapezoidal voltage waveform is present (fig. 5), with constant duty ratio and constant frequency $(20 \mathrm{kHz}-50 \mathrm{kHz}$, depending on the power rating). The device switching is performed synchronously at zero voltage. The 3 current generators are connected to isolated secondaries of the same transformer.

The trapezoidal waveform of the voltage and zero voltage switching of all semiconductor devices are required to minimize the amplitude, and to limit the upper frequency, of noise transmitted to the mains network. Zero voltage switching has also an important influence in the reduction of device power losses and system efficiency.
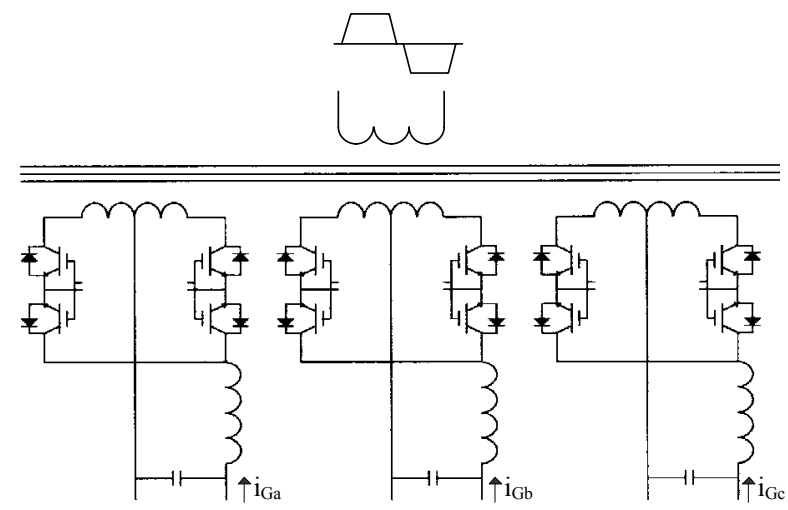

Fig. 5. The 3 parallel current generators connected to a single hf transformer.

The voltage at the input of the cycloconverter filter is modulated by integer pulses, in order to reduce switching losses. The inconvenience of this modulation process, over the traditional PWM, is the increase of filter volume and the consequent limitation of bandwidth. As the softswitching technique, used in the converters, permits frequencies for power pulses of $25 \mathrm{kHz}$, up to $50 \mathrm{kHz}$, depending on power, and the filter bandwidth is usually limited to the $25^{\text {th }}$ harmonic of mains frequency, integer pulse modulation is not an important drawback and remains a very good technical solution.
The transformer secondaries are designed for the maximum output peak power of the 3 current generators. However, in the primary of the transformer the addition of the instantaneous power in the 3 current generators is present. This implies a current with an amplitude strongly lower than 3 times the secondary amplitudes, also verified even in very harsh distortion conditions, due to the fact that currents in the parallel current generators are only the harmonic components (due to the complete cancellation of the fundamental components).

The series current generators can be designed as the parallel current generators (fig. 5). However, that configuration presents some drawbacks. The output voltage of the series current generators is typically $1 / 10$ of the nominal voltage. In spite of this low output voltage, the transistors in the cycloconverter must afford the peak value of the line voltage, in order to avoid disruption when the converter is shut off. And those

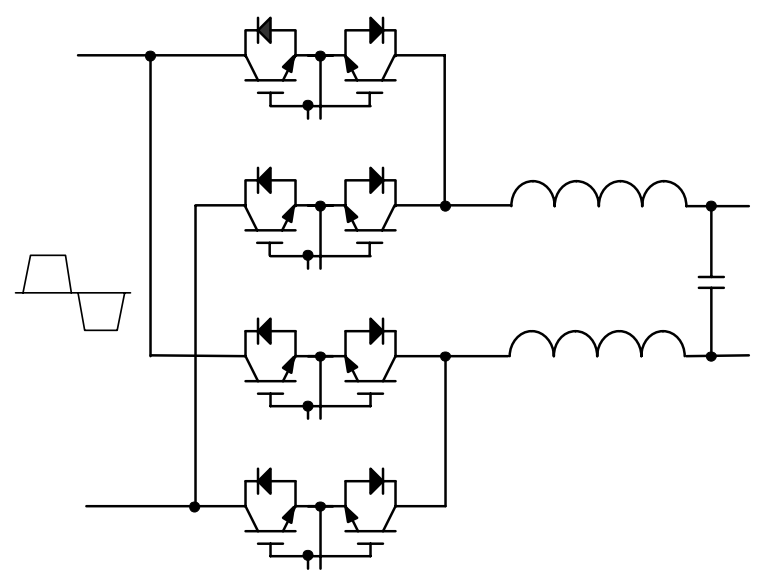

Fig. 6. Power converter for the series current generators associated to the line frequency transformers.

transistors shall conduct the total nominal input current in each phase. Consequently, the topology configuration of the Fig. 5 must be designed for a power level equal to the nominal input power. In high power systems it is less expensive to introduce 3 single phase transformers (Fig. 4) and use power converters rated at $1 / 10$ of nominal input power (Fig.6). Those transformers are bulky, because they operate at the line frequency and the secondary current is equal to the filter input current. However, the rated power remains very low, due to the fact that secondary voltage is the voltage difference from the input to the output filter voltages. The switching process in the converter of Fig. 6 requires a zero voltage at input to avoid important switching transients and overvoltages.

\section{Soft-Switching DC-AC Converter}

The soft-switching DC-AC converter of Fig. 7 presents 3 main sections:

- the storage capacitor of the active power filter $\left(\mathrm{V}_{\mathrm{DC}}\right)$;

- the soft-switching cell, performed by the transistors $S_{1}$ to $\mathrm{S}_{3}$, diodes $\mathrm{D}_{1}$ to $\mathrm{D}_{3}$, inductor $\mathrm{L}$ and capacitor $\mathrm{C}$; 
- a full-bridge DC-AC converter.

The soft-switching process, called switching input supply (SIS), permits the zero voltage switching of all transistors in the bridge circuit and an output voltage with a moderate value of $\mathrm{dv} / \mathrm{dt}$. Zero voltage switching is important to reduce the switching losses of the devices and to eliminate switching noise and spikes. The reduction of the switching noise and the moderate value of $\mathrm{dv} / \mathrm{dt}$ in the voltage output are very important to reduce the electromagnetic interference (radiated and conducted noise to the line network) [4]. The trapezoidal waveform is almost independent of the load conditions and presents a small time interval where voltage is zero.

The cycloconverters of the six current generators operate synchronously and all switching operations occur at zero voltage.

The analysis and design procedures of this converter are somewhat long and exceed the scope of this paper [5]. However it is convenient to understand the operation process.

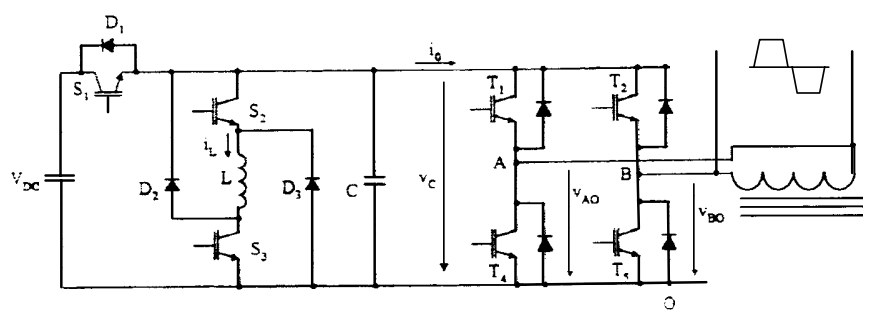

Fig. 7. Soft-switching DC-AC converter

The basic SIS circuit topology is shown in Fig. 8. The auxiliary switching circuit is performed by transistor $\mathrm{S}_{1}$, diode $\mathrm{D}_{1}$ and capacitor $C$.

Considering $i_{P}>0$ and transistor $\mathrm{S}_{1}$ on, the DC voltage source, $V_{D C}$, is connected to the converter, and supplies the load current to the bridge converter. Consequently, the SIS circuit and the converter circuit behave like a conventional voltage sourced inverter (VSI), since $v_{P}=V_{D C}$.

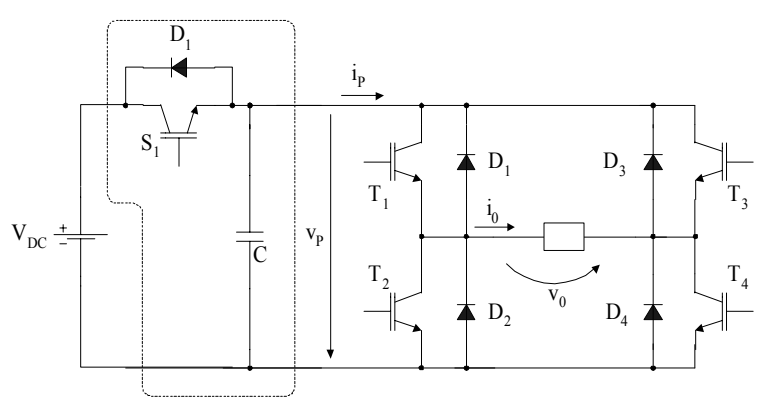

Fig.8. Basic SIS topology.

The main objective of the SIS circuit is the annulment of voltage $v_{P}$, whenever the main converter transistors need to be switched. Preceding any transition in the bridge switches, transistor $\mathrm{S}_{1}$ is turned off, allowing the discharge of capacitor $C$ to the inductive load of the converter. When the DC link voltage $v_{P}$ becomes zero, the voltages $v_{C E}$ in all converter switches are also at zero voltage, allowing lossless switching. After transitions in the bridge circuit, it is necessary to charge again the capacitor $C$ up to $v_{P}=V_{D C}$, in order to provide zero voltage switching turn-on of the transistor $\mathrm{S}_{1}$. The charge of capacitor $C$ results from the inversion of the current $i_{P}$ $\left(i_{P}<0\right)$, due to the inductance of the converter load and free-wheeling conduction of the bridge diodes, that occurs after transistor turn-off in the bridge circuit. Consequently, it is necessary to have an amount of stored energy in the load larger than the energy required to charge the capacitor up to $V_{D C}$. When $v_{P}=V_{D C}$, diode $\mathrm{D}_{1}$ turns on and $\mathrm{S}_{1}$ can be switched on at zero voltage. Once again, the voltage source $V_{D C}$ is connected to the inverter and the circuit returns to the initial operation.

The method described to charge the capacitor $C$ is dependent on the energy stored in the load. For instance, if we have a transformer it is necessary to guarantee that the magnetising energy is larger than the energy required to charge the capacitor. The charging and discharging times of capacitor $C$ are load dependent. For light loads those times can be very long, compromising the useful range of duty-ratio. The charging and discharging times are also important in terms of radio frequency spectrum, radiated by the converter. It is interesting to have long transition times, in order to cancel noise at the higher bands of frequency. However, the consequent duty-ratio limitations are undesirable. In high power applications it is interesting to manage jointly the maximum duty-ratio limit and noise cancellation. This objective may be achieved imposing a constant time for the capacitor charging (and discharging), independent of the load current. Using an auxiliary energy storage element (Fig. 9.) and controlling the value of the stored energy, it is possible to impose almost constant charging and discharging times enabling the operation with zero voltage switching, whenever the energy stored in the load is not sufficient to charge the capacitor.

The above objectives can be achieved by using the auxiliary switching circuit shown in Fig. 9. In this circuit, the switching process is independent of the load current as it will be shown. The auxiliary inductance stores the energy required for charging the switching capacitor. When $i_{P}<0, \mathrm{D}_{1}$ is on and $\mathrm{S}_{1}$ is off, the discharge of the

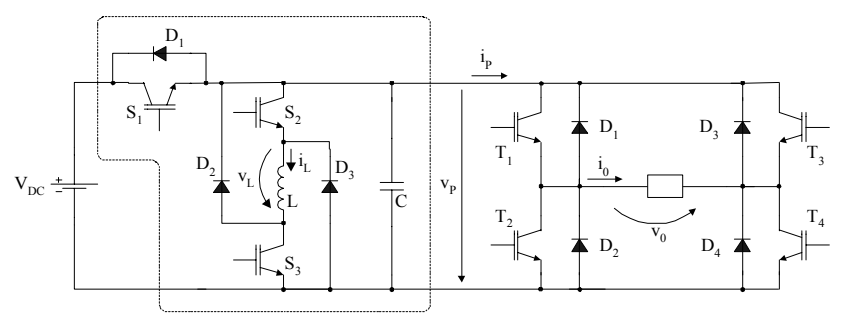

Fig.9. SIS topology with 3 auxiliary switches in a full bridge converter.

capacitor $C$ is provided by turning on $\mathrm{S}_{2}$ and $\mathrm{S}_{3}$, simultaneously. The current $i_{L}$ increases linearly (since $\left.v_{L}=V_{D C}\right)$ until the capacitor is completely discharged, i.e. 
$v_{P}=0$. Consequently the inverter transistors switch at zero voltage. Afterwards, it is necessary to charge the capacitor $C$, by turning off $\mathrm{S}_{2}$ and $\mathrm{S}_{3}$. The current $i_{L}$ flows through the diodes $\mathrm{D}_{2}$ and $\mathrm{D}_{3}$, and the energy stored in the inductor $L$ is transferred to the capacitor, until $v_{P}=V_{D C}$, and the circuit returns to the initial conditions.

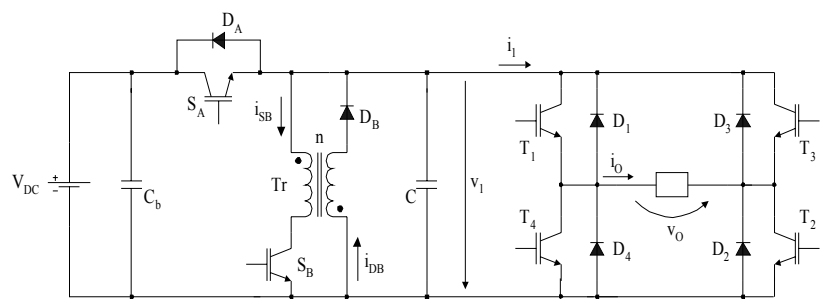

Fig. 10. SIS topology with two auxiliary switches (flyback configuration)

Figure 10 shows an alternative circuit for auxiliary energy storage, with the same operating principle, using a smaller number of semiconductor devices and a two windings inductor, in a configuration similar to a flyback converter. The circuits presented in Fig. 7., Fig. 9. and in Fig. 10. are equivalent.

The SIS concept has been verified in a prototype. The circuit ratings are DC link voltage $\mathrm{V}_{\mathrm{DC}}=500 \mathrm{~V}$, load current 2A, switching frequency $20 \mathrm{kHz}$. The main components are $\mathrm{L}=740 \mu \mathrm{H}, \quad \mathrm{C}=12 \mathrm{nF}, \quad$ IGBTs IRG4PC30UD and diodes STTA2006P. One of the major advantages of the SIS circuit is the inherent control of the $\mathrm{dv}_{\mathrm{C}} / \mathrm{dt}$ value, reducing EMI. It is possible to see in Fig. 11 a value of $\mathrm{dv}_{\mathrm{C}} / \mathrm{dt}$ lower than $500 \mathrm{~V} / \mu \mathrm{s}$.

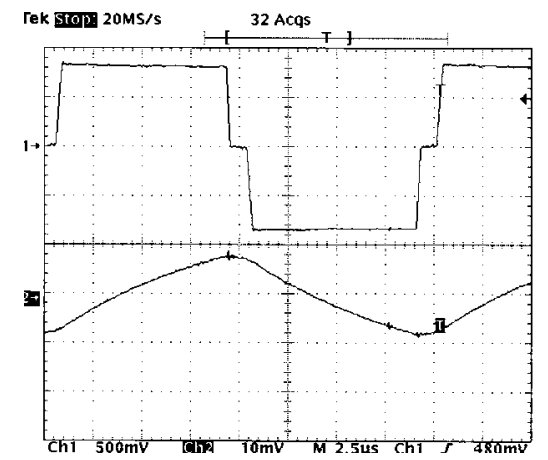

Fig. 11. Output voltage and current waveforms of the DC-AC soft-switching converter $(250 \mathrm{~V} / \mathrm{div}, 2 \mathrm{~A} / \mathrm{div}, 2.5 \mu \mathrm{s} / \mathrm{div})$

\section{Operating principles of the control circuits}

The hf DC-AC converter has no control functions over the output variables, because output voltage presents constant frequency, amplitude and duty ratio. It has only a local control of the magnetic energy stored in the inductor of the soft-switching cell and also some logic circuitry for transistors gating.

As referred previously, the control circuits of the series and parallel current generators are completely independent and also phase independent. This approach has several advantages, namely:

- the control and stability conditions are more accurate, reliable and easier, specially in transient conditions, and, consequently, it is possible to minimize the storage capacitor;

- load imbalance does not affect the filter operation and, consequently, it is possible to minimize the power rating of the power converters;

- line voltage imbalance does not affect the filter operation and, consequently, it is possible to achieve an almost sinusoidal input current;

- The effects of distortion generated by single-phase loads over the neutral currents are very well compensated, reducing the neutral current to its first harmonic, depending only on the load imbalance and power factor correction.

The disadvantage is an apparent higher complexity of the control circuitry, due to the multiplication of independent control circuits.

The parallel current generators control the filter output voltages (phase-neutral). The DSP circuit (Fig. 12) computes the amplitude of the first harmonic of the input voltage, in each period, and uses it as the reference value for the output voltage in the next period. This reference voltage is a sinusoidal waveform, in phase with input voltage and with amplitude proportional to the first harmonic of input voltage. The same calculation, over the output currents, is performed to obtain the 3 sinusoidal reference voltages for the series current generators.

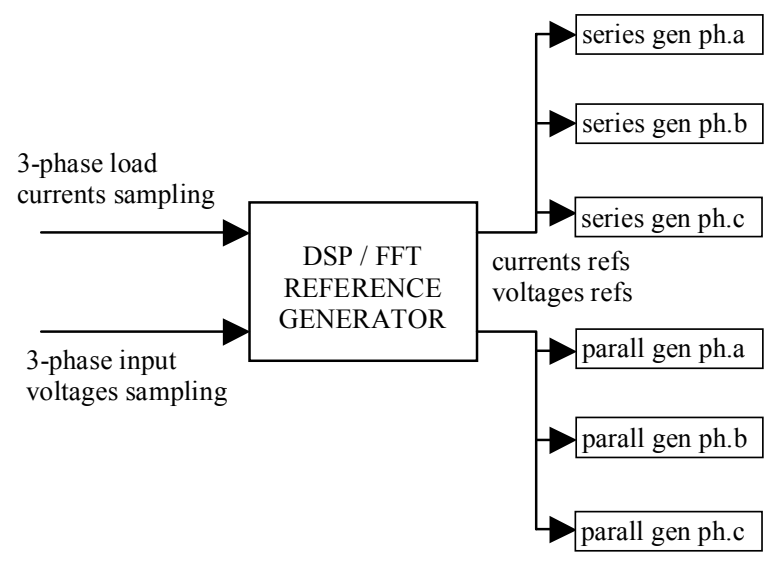

Fig. 12. DSP based reference generators.

The parallel current generator modulator is a current mode controller using a double prediction scheme for integral pulse modulation [6]. The predictor uses the instantaneous value of the error voltage and its time derivative to predict the required value for the current, in the output filter inductor, one period later. It computes also the estimated values of current for 3 different switching conditions for the voltage at the input of the 
LC filter of the cycloconverter $(+\mathrm{V}, 0,-\mathrm{V})$. The switching decision is adopted for the minimum difference between predicted error and predicted current. Cycloconverter switching, accordingly the decision, takes place in the next time interval where the trapezoidal waveform is at zero voltage.

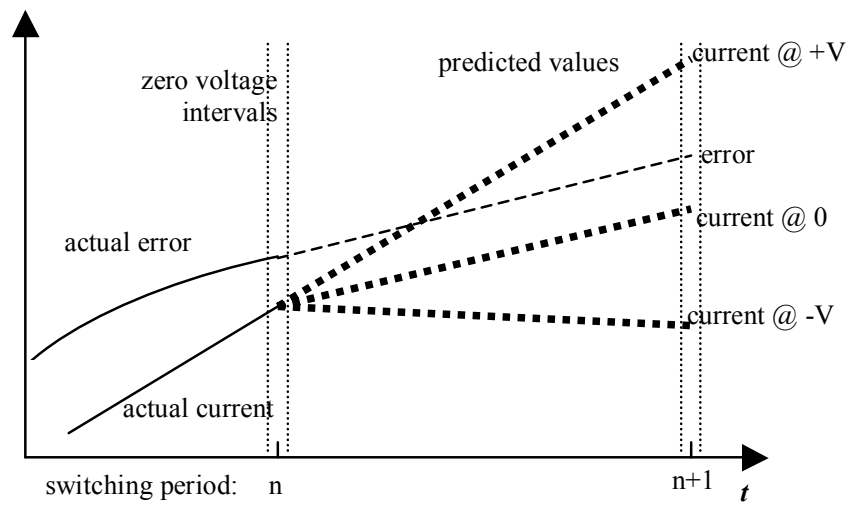

Fig. 9. Integer pulse modulation based on error minimization with predicted variables.

The control process of the series converter is more complex due to the requirement of control for the storage capacitor voltage. The conventional control process for the storage capacitor voltage is to divide the current reference signal by an error signal, resulting from the comparison of the capacitor voltage with a reference value. The method introduces distortion in the input current, if the measurement of capacitor voltage is continuous, during all the line frequency period. An alternative method proposed in $[7,8]$, the Unified Constant-frequency Integration Control (UCI) provides a good solution for PWM converters and it can be applied to integer pulse modulation, using long decision periods (20 to 100 switching periods). Such long decision periods introduce an important reduction of bandwidth of the current generator. In order to avoid this drawback of UCI in integer period modulation, the authors developed an alternative to the conversion of PWM to integer period modulation. In each switching period, is generated a PWM pulse resulting from the sampling of current error and capacitor voltage. This pulse is evaluated in order to measure the duty ratio. Based on the measured value of duty ratio in one period, the decision for the switching state in the next period is taken. The values of duty ratio in the intervals $(0,1 / 3),(1 / 3,2 / 3)$ and $(2 / 3,1)$ correspond to the state $-\mathrm{V}, 0$ and $+\mathrm{V}$ at the cycloconverter output. This process has a delay of one switching period.

\section{Conclusion}

The authors introduced a design process for series parallel power active filters that minimizes the power rating of the power converters, based on six power converters coupled to a single full-bridge soft-switching inverter. This inverter provides zero voltage switching for all the switching devices in all converters of the active power filter and limits the time derivatives of the voltages in all converters. This limitation of voltage derivatives and zero voltage switching are important features for the control of EMI.

New control strategies for active power filters have also been introduced, namely the instantaneous feedback and control for all the variables, with an independent control, in the 3 phases, of the line currents and output voltages.

\section{Acknowledgement}

Research project OCAP funded by Fundação para a Ciência e Tecnologia.

\section{References}

[1] V. Soares, P. Verdelho, "An Instantaneous Active and Reactive Current Component Method for Active Filters", IEEE Trans. on Power Electronics, vol. 15, pg. 660, July 2000.

[2] S. Mariethoz, A. C. Rufer, "Open Loop and Closed Loop Spectral Frequency Active Filtering", IEEE Trans. on Power Electronics, vol. 17, pg. 564, July 2002.

[3] Victor Anunciada, João Santana, "A New Configuration of Low Cost Rotative Diesel UPS System" IEEE International Telecommunications Energy Conference, INTELEC 96, Boston, USA, October 1996.

[4] D. M. Divan and G. Skibinski, "Zero-Switching-Loss Inverters for High-Power Applications", IEEE Tran. Industry Applications, Vol. 25, no. 4, pp. 634-643, July/August 1989.

[5] R. D. Monteiro, A V A Anunciada, "Design Principles of a Soft-Switching Cell Usable in Bridge DC-DC and DC-AC Converters", PESC 02, Cairns, Australia, Juin 2002, p. 10-2-1

[6] J. Fernando Silva, Beatriz V. Borges, Victor Anunciada, "Improving Control Strategies for HF Resonant Link Converters: The Current Mode Predictive Modulator", Procc. IEEE, Power Electronics Specialists Conference, PESC'91, Boston EUA, Juin 1991.

[7] L. Zhou, K.M. Smedley, Unified Constant-frequency Integration Control of Active Power Filters, $15^{\text {th }}$ APEC, New Orleans, Feb. 2000

[8] Z. Lai, K.M. Smedley, "A General Constant Frequency Pulse Width Modulator and Its Applications", IEEE Trans. On Circuits and Systems I: Fundamental Theory and Applications, vol.45 no. 4, IEEE, April 1998, pp 386-396 